\title{
Enhancing dimensional accuracy of FDM based biomedical implant replicas by statistically controlled vapor smoothing process
}

\author{
Jasgurpreet Singh Chohan ${ }^{1} \cdot$ Rupinder Singh ${ }^{2}$
}

Received: 29 June 2015/Accepted: 2 April 2016/Published online: 15 April 2016

(C) Springer International Publishing Switzerland 2016

\begin{abstract}
The aim of the present study is to highlight the benefits of vapor smoothing on ABS replicas prepared by fused deposition modeling (FDM) for rapid casting of biomedical implants. The FDM process induced stair steps on circular and sloping surfaces, which increased part dimensions when measured and led to oversized parts (contrary to reduced part dimensions in linear surfaces).The process originally developed for surface finishing employs the technique of plastic reflowing of heated material, in contrast to material removal in traditional finishing processes. Measurements performed on CMM helped in evaluating the dimensional tolerances of replicas which emerged to be within limits for three cycles of vapor smoothing (each of $20 \mathrm{~s}$ ). Similar parameter settings yielded the best surface finish, which supported the assumption of layer resettlement of the semi-liquid ABS material. The statistical analysis strongly indicated the vapor smoothing process to be statistically controlled.
\end{abstract}

Keywords Vapor smoothing - Fused deposition modeling · Hip prostheses · Dimensional accuracy

Rupinder Singh

rupindersingh78@yahoo.com

Jasgurpreet Singh Chohan

jaskhera@gmail.com

Punjab Technical University, Kapurthala 144601, India

2 Production Engineering Department, GNDEC, Ludhiana 141006, India

\section{Introduction}

Additive or layered manufacturing is a rapidly emerging class of advanced production techniques where products are manufactured by forming plastic material from 3D model data. It eliminates the traditional subtractive processes, use of jigs, fixtures, etc. A lesser sensitivity to geometric complexity, shorter lead time and greater flexibility is one of the key characteristics of these additive manufacturing techniques [1]. Fused deposition modeling (FDM), an additive manufacturing technique introduced in 1987 by Stratasys, has attracted more and more interest of researchers and industries due to simplicity. The highly complicated parts can be built within few hours by importing the CAD data in FDM machine where heated nozzle extruded the semi molten plastic material (generally ABS) layer by layer on frictionless table. The numerically controlled nozzle head moves in $x-y$ direction to deposit layer of desired shape and table lowers for new layers to be deposited on previous one [2].

The application of FDM has been extended in rapid casting techniques where replicas are prepared and finally casted via investment casting (IC) method. IC process has an ability to produce intricate shapes with high accuracy, surface finish, and thus, considered one of the most versatile metal casting processes [3]. The design of replicas can be customized as per requirement within very short span of time by editing CAD file which make it highly flexible.

The rapid casting via FDM has been successfully applied for medical applications [4] to manufacture geometrically complicated physical prototypes of human anatomy derived from scanned data. The process could accelerate the production of patient specific implants within lower costs and time. The image of defected bone 
retrieved from computerized tomography or magnetic resonance imaging can be transferred into 3D model which can be fabricated as replicas, and finally highly precise casting can be prepared [5].

The challenge of poor surface finish and dimensional accuracy of FDM parts still persists [6], and thus, parts require post processing before initiating casting process. The surface roughness and dimensional variability as compared to CAD data are the inherent defects of FDM which arises due to conversion and tessellation of CAD file. The STL (standard triangulation language) format approximates the surface as web of triangles which represent the part surface in place of plane curves which reduced the resolution. This gives rise to chordal defect which created difference between original CAD surface and triangle of tessellated model which further results in dimensional variability [7]. The small details, circular sections (Fig. 1) and thickness of part are highly affected and made oversized which would require post processing. The surface roughness occurs on part surface as each layer of thermoplastic is deposited on previous layer [8].

A lot of research work has been executed to reduce the surface roughness of FDM parts. Generally, post processing with material removal method is adopted (mechanical and vibratory finishing) which yielded good results, but resulted in poor dimensional accuracy, edge cutting and rounding at higher machining durations [2,9]. Moreover, material removal is not uniform when intricate and complex designs are finished which induces dimensional variability.

Thus, in addition to pre processing errors like: tessellation, mathematical approximations at CAD stage and post processing further increase the dimensional variability [10]. A lot of researchers have optimized FDM pre processing parameters and found that layers deposited at

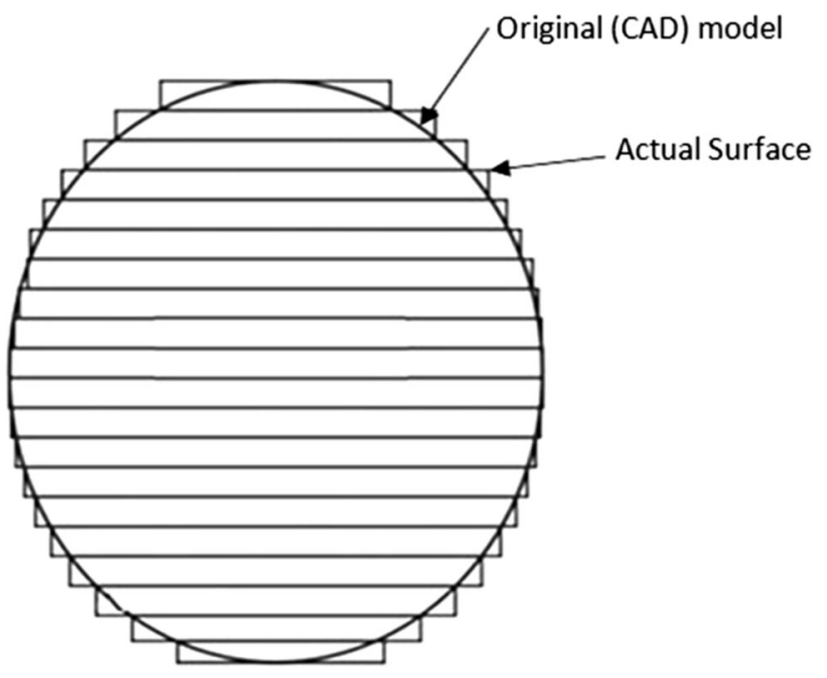

Fig. 1 Dimensional variation in actual surface achieved by FDM compared to CAD model angles $0^{\circ}$ and $90^{\circ}$ extracted minimum average surface roughness. The reduction in layer thickness enhanced both surface finish and dimensional accuracy but increased manufacturing time and cost [11]. Dyrbus [12] tested the dimensional accuracy of FDM for two test parts and it was found that circular shapes are manufactured oversized in contrary to reduced linear dimensions. Nanchariah et al. [13] found major impact of air gap and layer thickness while least significance of raster angle for enhancing the dimensional accuracy. Bakar et al. [8] measured that minute details and circular shapes of FDM parts are dimensionally worst affected with maximum deviation up to $1.9 \mathrm{~mm}$. Budzik [14] compared the influence of orientation angle on FDM and stereolithography, and found that curvilinear surfaces are more sensitive to dimensional variation even for lower layer thickness settings while FDM parts comparatively demonstrated higher accuracy. El-Katatny [15] checked the accuracy of FDM process for fabrication of actual human skull models and found undersized replicas with average deviation of $0.24 \%$ and standard deviation of $0.16 \%$. Bansal [16] optimized the impact of three process parameters via genetic algorithm and suggested $1.44 \mathrm{~mm}$, orientation angle $30^{\circ}$ and raster angle $60^{\circ}$ as best for achieving the best accuracy. Generally, the parts negatively deviated from CAD dimensions for length and width of standard rectangular specimen while positively deviated for thickness. Various authors have reported optimization of different process parameters to achieve dimensionally accurate products, but results may vary for the parts having complex shapes which would require adaptive slicing and use of algorithms [1]. Thus, surface finish and dimensional accuracy are two defects which are very difficult to eliminate together by optimizing FDM pre processing parameters.

The acetone exposure technique has been implemented by Galantucci et al. [17] by immersion of rectangular shaped test specimen in $90 \%$ dimethylketone and $10 \%$ water for $5 \mathrm{~min}$. The surface roughness was significantly reduced with less than $1 \%$ shrinkage in linear dimension. The acetone exposure erodes the material from upper surface, and thus, small weight loss occurs when parts are dried. Kuo and Mao [18] exposed FDM parts to acetone vapors and found excellent surface finish, minimal dimensional variation and increase strength. Similar results have been found by Garg et al. [19] in case of different geometrical features with variable orientation angles.

An advanced surface finishing technique has been developed by Stratasys [20] where hot chemical vapors tend to smoothen the surface of parts without an actual contact of tool with surface. The maiden study has been performed which reported very smooth surface with minimum impact on dimensional accuracy on standard Grimm test parts [21]. The vapor smoothing can be implemented to 
enhance surface finish of biomedical implant replicas, but it is required to check the impact of vapors on actual replicas as results may differ due to complex design. Thus, to recommend the vapor smoothing process for biomedical implant production, it is required to investigate the dimensional tolerances of actual replicas undergone smoothing process.

In present study, the hip prostheses have been prepared at parameters to yield the best dimensional accuracy [8, 13], and finally treated with hot vapors. The impact of different process parameters has been studied followed by statistical analysis of vapor smoothing process. The deviation of part dimension from original CAD surface had been inspected as per industrial requirements in terms of international tolerance grades.

\section{Experimental setup}

The hip prosthesis has been selected as a benchmark to evaluate the dimensional tolerances of vapor smoothed FDM parts as compared to CAD model. The specimen of hip prostheses (Fig. 2) has been designed in "Solidworks 2014" and converted into.STL format. This file was processed through CatalystEx software which slices and automatically generates tool paths for FDM system. The commercial FDM of 'Stratasys USA, Model uPrint-SE' has been used to fabricate the replicas at best input parameters to yield the best dimensional accuracy and surface roughness. In the first stage, eighteen replicas (in the set of $2 \times 9$ ) have been prepared keeping constant FDM parameters, i.e., layer thickness $0.254 \mathrm{~mm}$, orientation angle $0^{\circ}$, high density, $0^{\circ}$ raster angle with no air gap. Out of these, nine samples (in 02 sets) have been used to carry out preliminary investigations to identify the best smoothing parameters. The parameters have potential to

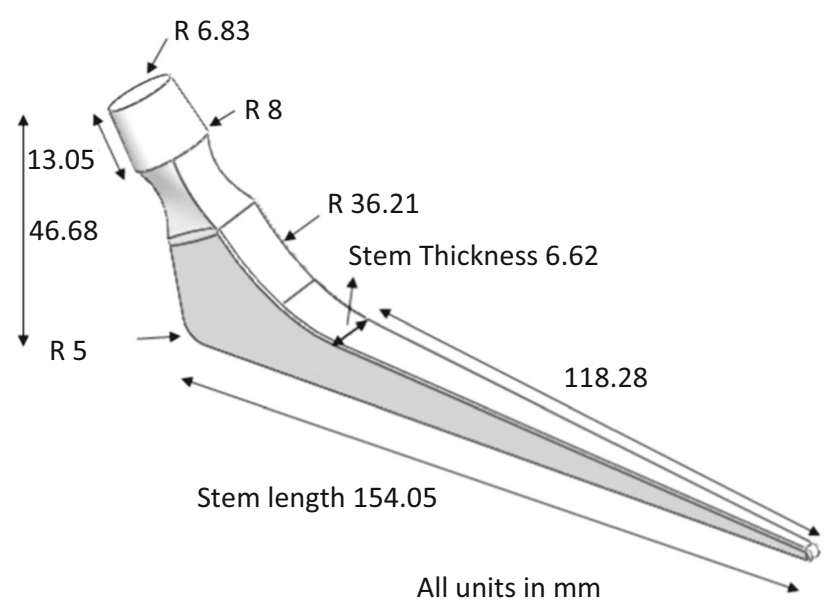

Fig. 2 Isometric view of hip prostheses (benchmark) control the dimensional accuracy within limits, but the assumption is invalid while dealing with complicated designs and geometry. The hip prostheses has varying stem thickness along the length, and thus, base table has to frequently move in downward direction for deposition of layers which induced stair steps on stem surface.

The sloping down stair steps can be clearly seen from left to right in transverse view of stem section (in Fig. 3). The rectangular portion highlighted in Fig. 3 indicated the variation between CAD model (represented with straight path) and actual surface (represented with stepped path). The stair steps can even be identified from the top view of Fig. 3 .

The vapor smoothing has been performed on "Finishing Touch Smoothing Station" (Fig. 4) having two chambers, i.e., cooling and smoothing chamber $(330 \times 406 \times$ $508 \mathrm{~mm}$ ). The vapor smoothing apparatus operates with smoothing fluid which is highly volatile (boiling point $43{ }^{\circ} \mathrm{C}$ ) with composition of Decafluoropentane (30-30 \%) and Trans-Dichloroethylene (70 \%).

The smoothing fluid is heated in smoothing chamber to $65{ }^{\circ} \mathrm{C}$ at which this volatile fluid vaporizes and start to rise until it reaches the condensation zone. In condensation zone, the fluid again cools down and recirculated. The hot vapors enter the surface of ABS parts and permanently reflows the plastic material to fill the rough steps. The parts are required to be hanged in smoothing chamber for few seconds and afterwards cooled in cooling chamber for few minutes. The cooling chamber is cooled by cooling the coils connected to refrigeration unit of maintain $0{ }^{\circ} \mathrm{C}$ temperature where part surface is settled after heating. The parts are required to be cooled (pre-cooling) for few minutes before smoothing process to achieve the best results [21]. The pneumatic lid of smoothing chamber is normally closed; controlled by foot switch to avoid the exit of vapors and only opened when parts are needed to be placed or taken out.

It is required to investigate the effect of different input parameters of vapor smoothing apparatus on dimensional tolerances but various temperatures and pressures cannot be varied. The cooling and smoothing durations are only controllable parameters out of which smoothing time has major impact as vapors interact with part surface at that stage. It is recommended by manufacturer that the precooling smoothing post-cooling cycle can be repeated again and again till the desired results are achieved [22]. Thus, an experimentation log has been designed to verify the influence of smoothing time $(t)$ and number of cycles (C) on dimensional accuracy. Three smoothing time (10, 15 and $20 \mathrm{~s})$ and three cycles $(1,2,3)$ have been selected as control parameters while pre-cooling and post-cooling times are held constant (10 min each).

Thus, nine samples (prepared in 02 sets) have been used to evaluate the impact of each factor, i.e., smoothing time 
Fig. 3 Stair steps visible on part surface after fabrication via FDM

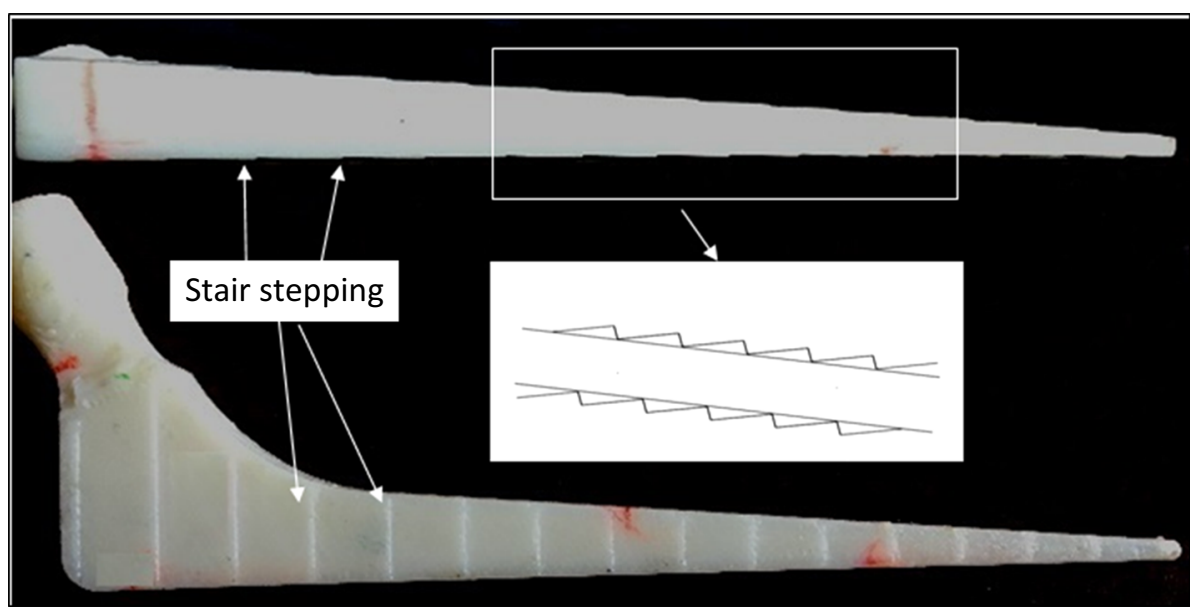

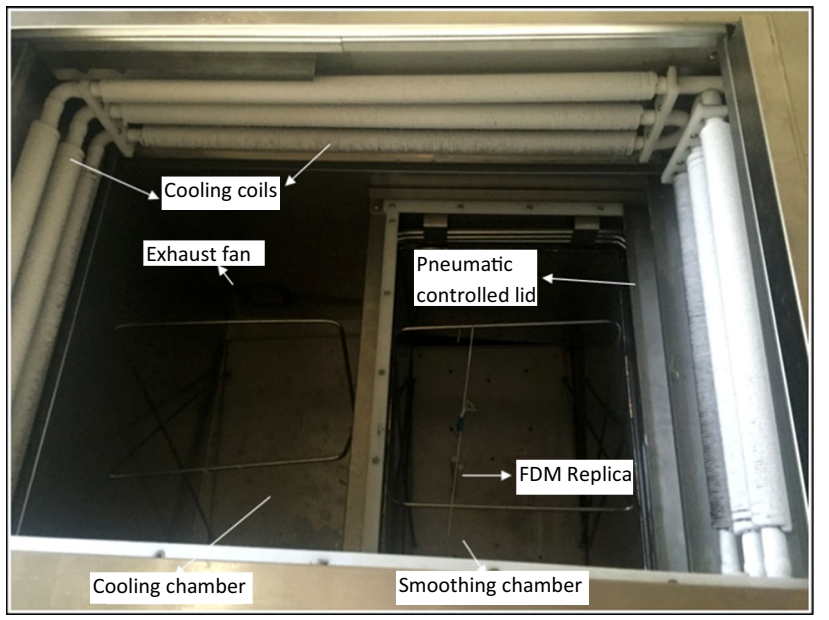

Fig. 4 Schematic of vapor smoothing process

and number of cycles (three levels each) on dimensional accuracy. The measurement path for external surface of benchmark has been generated through the computational software of GEOPAK v2.4.R10 coordinate measuring machine (CMM). The highlighted side (and its opposite) of specimen (Fig. 2) has maximum exposure (due to large surface area) to vapors which would have utmost impact on thickness. The thickness of replica may play a critical role during IC process, and thus, selected as target dimension for achieving precise casting. The measurement paths direct the movement of probe of CMM along the trajectories normal to surface of replica. The external surface has been measured by about 70 points. For each point, the software calculates the deviations between theoretical (CAD) and measured positions for $X, Y$ and $Z$ axis. The surface roughness has been measured by "Mitutoyo SJ210 " having stylus tip radius of $2 \mu \mathrm{m}$, tip angle $60{ }^{\circ} \mathrm{C}$ and measuring force of $0.75 \mathrm{mN}$. The measurements have been recorded employing Gaussian filter, cut-off length 0.25 and $2.5 \mathrm{~mm}$ exploratory length as per ISO 4287 regulations
[23]. The three measurements have been taken for each sample using surface roughness tester and mean has been taken as final value as shown in Table 1. Due to varying thickness along the length, the measurements have been taken at single location (118.28 $\mathrm{mm}$ from below on stem) for all the specimens and CAD model for comparison. The large variation in thickness of FDM replicas before smoothing (average $6.8642 \mathrm{~mm}$ ) was measured as compared to original CAD model thickness $(6.62 \mathrm{~mm})$.

\section{Analysis of results}

The measurements of thickness of stem section of hip prostheses along with average surface roughness (before and after smoothing) on the stem surface have been shown in Table 1 . There has been significant reduction in average surface roughness (average $82.74 \%$ ) of ABS replicas after vapor smoothing. The average thickness of replicas after smoothing is $6.7103 \mathrm{~mm}$ which reduced as compared to thickness before smoothing (average $6.8642 \mathrm{~mm}$ ). The vapor smoothing process proves to be more efficient and precise to preserve minute details of design as there is no actual contact with tool. In conventional finishing techniques like barrel finishing [2], there is a use of barrel finishing media on parts and as a result of this thin sections are under risk of breakage. Boschetto [24] reported improved surface finish with increase in barrel finishing time, but it increases material removal rate which manifested large dimensional variations on part surface. Fischer and Schoppner [9] suggested vibratory bowl grinding as mild finishing process without damaging edges and corners when performed at low intensity grinding but this practice reduce finishing efficiency. Thus, vapor smoothing process could prove cleaner, rapid and highly efficient finishing process in context of production, but it required to evaluate the impact of smoothing process on part surface and geometry using statistical tools. 
Table 1 Dimensional and surface roughness measurements for samples undergone vapor smoothing

\begin{tabular}{|c|c|c|c|c|c|}
\hline Sample no. & Smoothing conditions & Thickness $(\mathrm{mm})^{\mathrm{a}}$ & $\begin{array}{l}\text { Initial surface } \\
\text { roughness } R_{\mathrm{a}}(\mu \mathrm{m})^{\mathrm{a}}\end{array}$ & $\begin{array}{l}\text { Final surface } \\
\text { roughness } R_{\mathrm{a}}(\mu \mathrm{m})^{\mathrm{a}}\end{array}$ & $\begin{array}{l}\text { Percentage improvement } \\
\text { in surface finish }{ }^{\mathrm{a}}\end{array}$ \\
\hline 1 & $t=10 \mathrm{~s}, C=1$ & 6.8406 & 3.8012 & 1.1152 & 70.66 \\
\hline 2 & $t=10 \mathrm{~s}, C=2$ & 6.7905 & 3.8005 & 0.9010 & 76.29 \\
\hline 3 & $t=10 \mathrm{~s}, C=3$ & 6.7320 & 3.7994 & 0.6865 & 81.93 \\
\hline 4 & $t=15 \mathrm{~s}, C=1$ & 6.7268 & 3.8010 & 0.8738 & 77.01 \\
\hline 5 & $t=15 \mathrm{~s}, C=2$ & 6.6962 & 3.7998 & 0.6220 & 82.63 \\
\hline 6 & $t=15 \mathrm{~s}, C=3$ & 6.6607 & 3.8022 & 0.4459 & 88.27 \\
\hline 7 & $t=20 \mathrm{~s}, C=1$ & 6.6821 & 3.8038 & 0.6337 & 83.34 \\
\hline 8 & $t=20 \mathrm{~s}, C=2$ & 6.6520 & 3.8009 & 0.3808 & 89.98 \\
\hline 9 & $t=20 \mathrm{~s}, C=3$ & 6.6321 & 3.8026 & 0.2049 & 94.61 \\
\hline
\end{tabular}

${ }^{a}$ Average value of 9 samples prepared in 2 sets to reduce the experimental error

Table 2 Class of different IT grades according to UNI EN 20286-1 for samples

\begin{tabular}{|c|c|c|c|c|c|c|}
\hline \multirow[t]{2}{*}{ Sample no. } & \multirow[t]{2}{*}{ Smoothing conditions } & \multicolumn{2}{|c|}{ Thickness (mm) } & \multirow{2}{*}{$\begin{array}{l}\text { Standard tolerance } \\
\text { factor }(i)\end{array}$} & \multirow{2}{*}{$\begin{array}{l}\text { Tolerance } \\
\text { unit }(n)\end{array}$} & \multirow[t]{2}{*}{ IT grades } \\
\hline & & $T_{\mathrm{JN}}$ & $T_{\mathrm{JM}}$ & & & \\
\hline 1 & $t=10 \mathrm{~s}, c=1$ & 6.62 & 6.8406 & 0.85 & 259.5 & IT-13 \\
\hline 2 & $t=10 \mathrm{~s}, c=2$ & 6.62 & 6.7905 & 0.85 & 200.5 & IT-12 \\
\hline 3 & $t=10 \mathrm{~s}, c=3$ & 6.62 & 6.7320 & 0.85 & 131.7 & IT-12 \\
\hline 4 & $t=15 \mathrm{~s}, c=1$ & 6.62 & 6.7268 & 0.85 & 125.6 & IT-12 \\
\hline 5 & $t=15 \mathrm{~s}, c=2$ & 6.62 & 6.6962 & 0.85 & 89.6 & IT-11 \\
\hline 6 & $t=15 \mathrm{~s}, c=3$ & 6.62 & 6.6607 & 0.85 & 47.8 & IT-9 \\
\hline 7 & $t=20 \mathrm{~s}, c=1$ & 6.62 & 6.6821 & 0.85 & 73 & IT-10 \\
\hline 8 & $t=20 \mathrm{~s}, c=2$ & 6.62 & 6.6520 & 0.85 & 37.6 & IT-9 \\
\hline 9 & $t=20 \mathrm{~s}, c=3$ & 6.62 & 6.6321 & 0.85 & 14.2 & IT-7 \\
\hline
\end{tabular}

Thus, the output achieved from CMM in form of dimensional measurements has been utilized to calculate the tolerance unit $n$ that further derives standard tolerance factor $i$ as defined in standard UNI EN 20286-1 [25]. The standard tolerances corresponding to IT grades (IT-5 to IT18) for the linear dimensions (up to $500 \mathrm{~mm}$ ) have been evaluated considering standard tolerance factor $i(\mu \mathrm{m})$ given by the formula:

$i=\left(0.45 \times T^{1 / 3}\right)+(0.001 \times T)$

where $T$ is the geometric mean of the nominal thickness in $\mathrm{mm}$.

The standard tolerances are not separately evaluated for each nominal size, but for the whole range of nominal sizes. The number of tolerance unit $n$ for the nominal thickness $T_{\mathrm{JN}}$ has been evaluated as:

$n=1000\left(T_{\mathrm{JN}}-T_{\mathrm{JM}}\right) / i$

where $T_{\mathrm{JM}}$ is the measured thickness.

The tolerance has been expressed as the multiple of $i$, for example the grade IT-14 corresponds to $400 i$ with $n=400$. For each thickness value, the corresponding $u$ values has been calculated and taken as reference index for the evaluation of International tolerance grades. The classification of different IT grades as per UNI EN 20286-1 is shown in Table 2. The smoothing time of $23 \mathrm{~s}$ and three cycles lead to requisite tolerance limits, and thus, accepted for statistical analysis.

The increase in smoothing time and smoothing cycles is directly proportional in improving accuracy and surface finish of replicas. The heated vapors infiltrate the upper surface and ABS plastic material reflows temporarily and settles down evenly. The semi-liquid tend to achieve minimum area under the effect of surface tension forces, and thus, stair steps are considerably reduced as smoothing time and cycles are increased as shown in Fig. 5. This fact is supported by observations that dimensional accuracy of replicas has increased even after performing surface finishing operation. Generally, traditional surface finishing techniques cut away surface asperities, and thus, material removal takes place but there is no material loss in vapor smoothing process as material on upper surface upper is evenly settled down after cooling. This enhanced both surface finish and dimensional accuracy for oversized parts. 
(a)

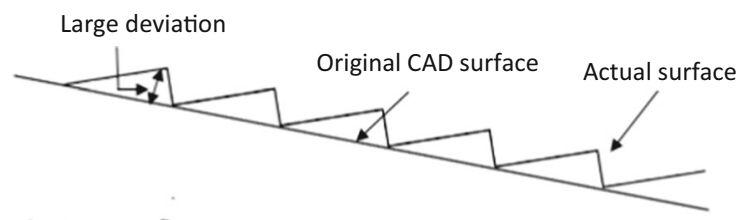

(b)

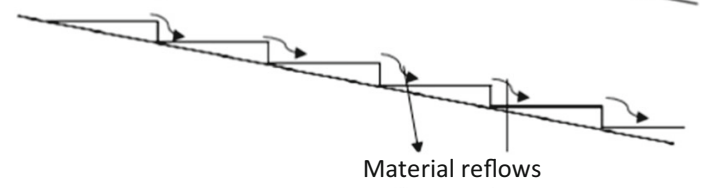

(c)

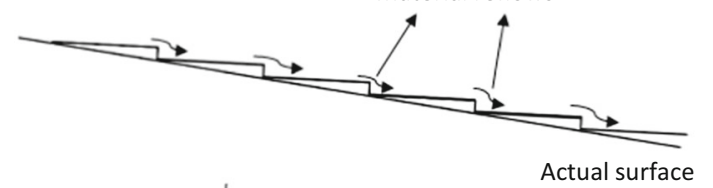

(d)

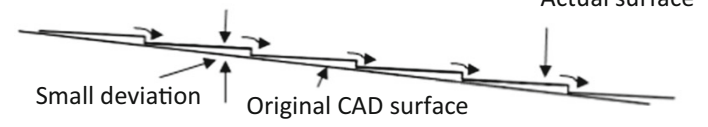

Fig. 5 Material reflow on part surface $\mathbf{a}$ before smoothing $\mathbf{b}$ smoothing $t=10 \mathrm{~s}, C=1 \mathrm{c}$ smoothing $t=15 \mathrm{~s}, C=2$ d smoothing $t=20 \mathrm{~s}, C=3$

Further (based on observations of Table 2), to ascertain the statistical consistence of vapor smoothing process, the six replicas have been treated at best vapor smoothing parameters $(t=20 \mathrm{~s}, C=3)$. After the measurements of thickness value at specific location with CMM, the dimensions have been measured (see Table 3). The surface finish and accuracy are acceptable for plastic replicas per requirements of casting industry which can be verified by SEM micrograph images (Fig. 6). The height of stair step has been significantly reduced after three vapor smoothing cycles of $20 \mathrm{~s}$ each. The run chart of measured values has been developed Fig. 7.

\section{Standard normal deviate $(Z)$ calculations}

It is a normally distributed random variable having zero mean and unit standard deviation generally employed to check the statistical independence of observed data. The theory of standardized variable [26] has been applied in present study to validate the effectiveness, consistency and accuracy of vapor smoothing process at specific input parameters $(t=20 \mathrm{~s}, C=3)$.

It is assumed that mean $(\mu)$ and standard deviation $(\sigma)$ of the population are normally distributed, and then the standard normal deviate $Z$ for the variable data $X_{i}$ is defined as:

$Z=\frac{X_{i}-\mu}{\sigma}$

The six confirmatory experiments for vapor smoothing has been executed for this purpose as discussed above, and thus, statistical analysis can be performed within limited number of experimental runs.

$E(\text { run })_{\mathrm{AB}}=\left(\frac{N}{2}+1\right)$

where $E$ (run $)_{\mathrm{AB}}$ is the expected number of run above and below is whereas $N$ is the number of observations.

$E(\text { run })_{\mathrm{AB}}=\left(\frac{6}{2}+1\right)=4$

The standard deviation above and below can be calculated as $\sigma_{\mathrm{AB}}=\sqrt{\left(N-\frac{1}{4}\right)}$

$\sigma_{\mathrm{AB}}=\sqrt{\left(6-\frac{1}{4}\right)}=1.118$

$Z_{\mathrm{AB}}=\left\{\operatorname{RUN}_{\mathrm{AB}}-E(\text { run })_{\mathrm{AB}}\right\} / \sigma_{\mathrm{AB}}$

where, $\mathrm{RUN}_{\mathrm{AB}}$ is the actual number of runs obtained above and below:

$Z_{\mathrm{AB}}=\{1-4\} / 1.118=-2.6834$

$\left|Z_{\mathrm{AB}}\right|=2.6384$

$E(\text { run })_{\mathrm{UD}}=(2 N)-\frac{1}{3}$

where $E(\text { run })_{\mathrm{UD}}$ is the expected number of runs up and down
Table 3 Benchmark thickness measurements for vapor smoothing $(t=20 \mathrm{~s}, C=3)$

\begin{tabular}{lllll}
\hline Experiment no. & Thickness $(\mathrm{mm})$ & Mean & Above or below of mean & Up or down \\
\hline 1 & 6.6314 & 6.6317 & $\mathrm{~B}$ & \\
2 & 6.6307 & 6.6317 & $\mathrm{~B}$ & $\mathrm{D}$ \\
3 & 6.6316 & 6.6317 & $\mathrm{~B}$ & $\mathrm{U}$ \\
4 & 6.6320 & 6.6317 & $\mathrm{~A}$ & $\mathrm{U}$ \\
5 & 6.6322 & 6.6317 & $\mathrm{~A}$ & $\mathrm{U}$ \\
6 & 6.6327 & 6.6317 & $\mathrm{~A}$ & $\mathrm{U}$ \\
Mean & 6.6317 & & Run $=1$ & $\mathrm{U}$ and D $=1$ \\
Standard deviation & 0.000695 & & & \\
\hline
\end{tabular}

$A$ above the mean, $B$ below the mean, $U$ up from previous reading, $D$ down from previous reading 

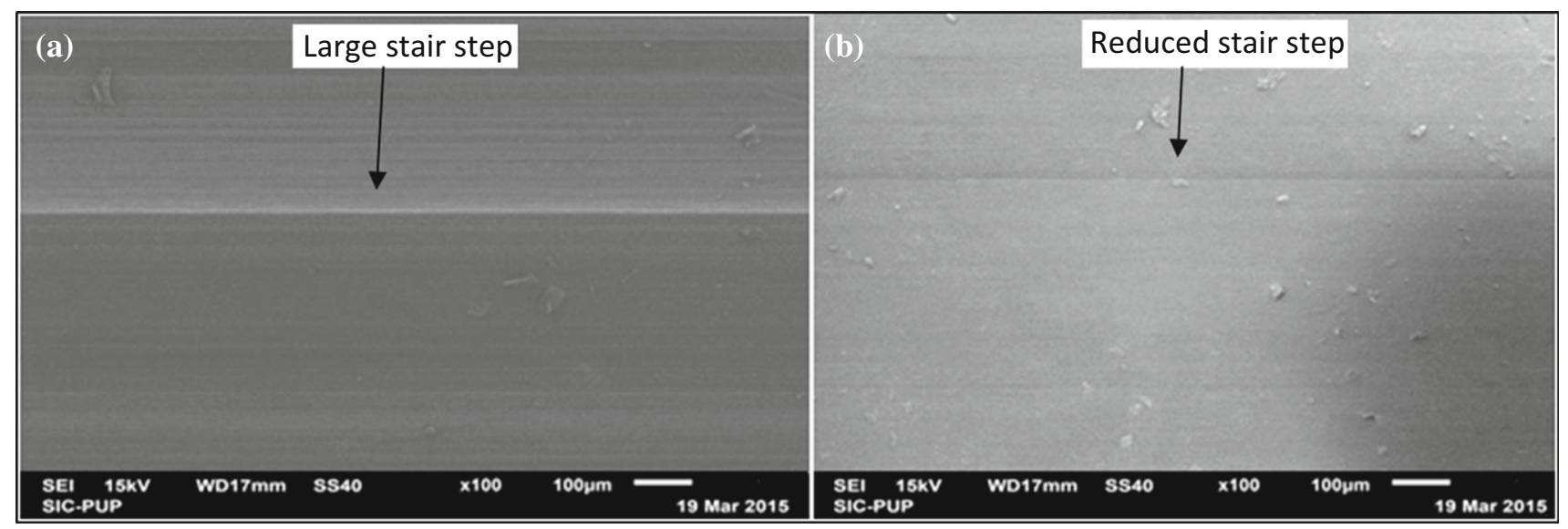

Fig. 6 SEM images of stair steps a before smoothing $\mathbf{b}$ after smoothing

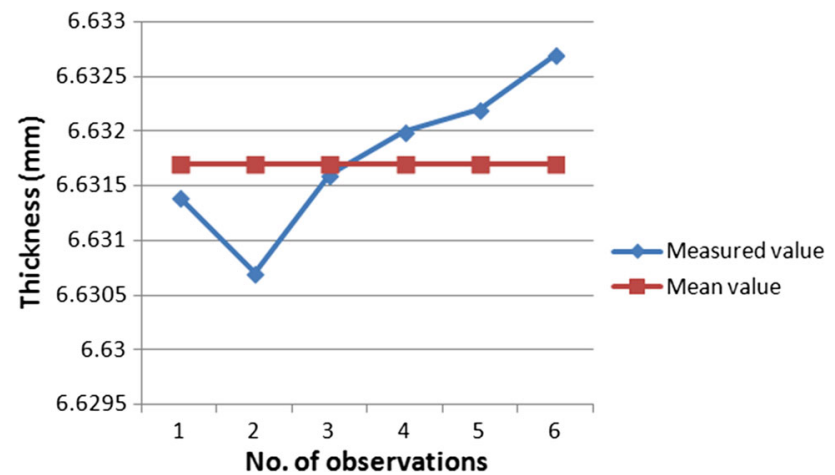

Fig. 7 Run chart for measured thickness of replicas

$$
\begin{aligned}
& E(\text { run })_{\mathrm{UD}}=(2 \times 6)-\frac{1}{3}=3.667 \\
& \sigma_{\mathrm{UD}}=\sqrt{(16 N)-(29 / 90)}
\end{aligned}
$$

where, $\sigma_{\mathrm{UD}}$ is the standard deviation up and down

$$
\begin{aligned}
& \sigma_{\mathrm{UD}}=\sqrt{(16 \times 6)-(29 / 90)} \\
& \sigma_{\mathrm{UD}}=0.8628 \\
& Z_{\mathrm{UD}}=\left\{\mathrm{RUN}_{\mathrm{UD}}-E(\text { run })_{\mathrm{UD}}\right\} / \sigma_{\mathrm{UD}}=(1-3.667) / 0.8628 \\
& Z_{\mathrm{UD}}=-3.091 \\
& \left|Z_{\mathrm{UD}}\right|=3.091
\end{aligned}
$$

The critical values for $Z$ are obtained using Microsoft Office Excel.

$$
Z_{\text {crit }}=\operatorname{NORMSINV}(1-\alpha / 2)
$$

Normally, the decision making is done with certain margin of error ' $\alpha$ ' and taken as equal to 0.005 i.e. there are only $5 \%$ chances of arriving at wrong conclusions. Thus, $Z_{\text {crit }}=1.959963$.

The relation between normal deviates for decision making is:
$\left|Z_{\mathrm{AB}}\right|>Z_{\text {crit }}$ and $/$ or $\left|Z_{\mathrm{UD}}\right|>Z_{\text {crit }}$

If the above relation is satisfied, them a non-random pattern exists in experimentation.

In the present observations, $\left|Z_{\mathrm{AB}}\right|$ and $\left|Z_{\mathrm{UD}}\right|$ are less than $Z_{\text {crit }}$ which indicates existence of non random pattern.

The normality of distribution has to be verified before drawing out any predictions or statistical conclusions. Even, after the collection of large data, it is difficult to superimpose the normal curve on the histogram. The minimum 50 observations are required to form a histogram; however, more would yield better results. But for fewer numbers of observations, it would become more cumbersome to access the normality of underlying distribution. The cumulative probability plot $\left(P_{\mathrm{i}}\right)$ is defined by equation: $P_{\mathrm{i}}=(\mathrm{S}$. no. -0.5$) / N$ where $\mathrm{S}$. no. is the serial number of observations aligned in ascending order, and $N$ is the total number of observations.

The standard normal deviate $Z=\frac{X_{i}-\mu}{\sigma}$

If $Z$ follows a normal distribution, that has mean $(\mu)=0$ and standard deviation $(\sigma)=1$, then:

$f(Z)=\sqrt{\frac{1}{2 \pi} e^{Z^{2} / 2}}$

The above equation follows the normal probability curve and data close to it also follows a normal probability curve. The standard normal deviate $(Z)$ has been calculated using cumulative probability and thickness of replicas have been arranged in ascending order (Table 4).

The normal probability curve has been plotted to predict the probability as shown in Fig. 8. The value of Pearson's coefficient $\left(R^{2}=1\right)$ indicates perfect correlation and the data fits very well to the trendline. The aforesaid data followed non random pattern and lied under normal probability curve. Even the $X$-bar and $R$-bar charts cannot be plotted due to lesser number of observations, but there lies 
Table 4 Standard normal deviate and thickness in ascending order

\begin{tabular}{llll}
\hline S. no & $\begin{array}{l}\text { Pi (cumulative } \\
\text { probability) }\end{array}$ & $\begin{array}{l}\text { Z (standard normal } \\
\text { deviate) }\end{array}$ & $\begin{array}{l}\text { Thickness } \\
(\mathrm{mm})\end{array}$ \\
\hline 1 & 0.083 & -1.43 & 6.6307 \\
2 & 0.25 & -0.43 & 6.6314 \\
3 & 0.416 & -0.143 & 6.6316 \\
4 & 0.583 & 0.43 & 6.6320 \\
5 & 0.75 & 0.719 & 6.6322 \\
6 & 0.916 & 1.43 & 6.6327 \\
\hline
\end{tabular}

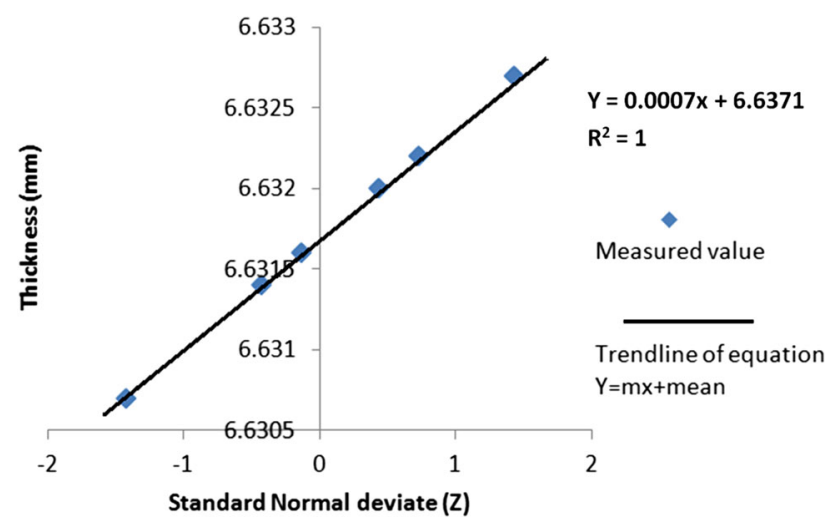

Fig. 8 Normal probability curve for benchmark

a very strong evidence that vapor smoothing process (at $t=20 \mathrm{~s}, C=3$ ) is statistically controlled and consistent.

\section{Conclusions}

The present research signifies the impact of vapor smoothing process for surface finishing besides maintaining the dimensions of ABS replicas as nearest to original (CAD), and thus, eliminates the drawback of dimensional variability in FDM parts. The replicas of hip prostheses satisfy the required industrial tolerances for thickness values when vapor treated three times for $20 \mathrm{~s}$ each. Then six replicas finished aforesaid vapor smoothing parameters strongly indicated the process to be statistically controlled as per 20286-1 (1995) ISO standards. Hence, the vapor smoothing of FDM based ABS replicas can be used for preparing investment castings of patient specific implants.

\section{References}

1. Yang Y, Fuh JYH, Loh HT, Wong YS (2003) A volumetric difference-based adaptive slicing and deposition method for layered manufacturing. Trans Am Soc Mech Eng J Manuf Sci Eng 125(3):586-594
2. Boschetto A, Bottini L, Veniali F (2013) Microremoval modeling of surface roughness in barrel finishing. Int J Adv Manuf Technol 69:2343-2354

3. Pattnaik S, Jha PK, Karunakar DB. A review of rapid prototyping integrated investment casting processes. J Mater Design Appl. doi: $10.1177 / 1464420713479257$ (ahead of print 2013)

4. Dhakshyani IR. Computer modelling of rapid prototyping models for hip surgery. In: Second International conference on technological advances in electrical, electronics and computer engineering, Kuala Lumpur, Malaysia, March 18-20 2014. pp. $156-163$

5. Winder J, Bibb R (2005) Medical rapid prototyping technologies: state of the art and current limitations for application in oral and maxillofacial surgery. J Oral Maxillofac Surg 6(7):1006-1015

6. Ippolito R, Iuliano L, Gatto A (1995) Benchmarking of rapid prototyping techniques in terms of dimensional accuracy and surface finish. CIRP Ann 44(1):157-160

7. Vasudevarao B, Natarajan DP, Henderson M. Sensitivity of RP surface finish to process parameter variation. In: Proceedings of 11th Solid Freeform Fabrication Symposium, Austin, USA, August 2000. pp. 252-58

8. Bakar NSA, Alkahari MR, Beojang H (2010) Analysis on fused deposition modelling performance. J Zhejiang Univ Sci A 11(12):972-977

9. Fisher M, Schoppner V. Finishing of ABS-M30 parts manufactured with fused deposition modeling with focus on dimensional accuracy. In: Proceedings of 25th Solid Freeform Fabrication Symposium 2014, Austin, USA. pp. 923-934

10. Cajal C, Jorge S, David S, Jesus V (2016) Efficient volumetric error compensation technique for additive manufacturing machines. Rapid Prototyp J 22(1):2-19. doi:10.1108/RPJ-052014-0061

11. Pérez CJL (2010) Analysis of the surface roughness and dimensional accuracy capability of fused deposition modeling processes. Int J Prod Res 40(12):2865-2881

12. Dyrbus G. Investigation of quality of rapid prototyping FDM method. In: 14th International conference on trends in the development of machinery and associated technology, Mediterranean Cruise, September 11-18; 2010. pp. 629-632

13. Nancharaiah T, Raju DR, Raju VR (2010) An experimental investigation on surface quality and dimensional accuracy of FDM components. Int J Emerg Technol 1(2):106-111

14. Budzik G (2010) Geometric accuracy of aircraft engine blade models constructed by means of the generative rapid prototyping methods FDM and SLA. Adv Manuf Sci Technol 34(1):33-43

15. El-Katatny I, Masood SH, Morsi YS (2010) Error analysis of FDM fabricated medical replicas. Rapid Prototyp J 16(1):36-43

16. Bansal R. Improving dimensional accuracy of fused deposition modelling (FDM) parts using response surface methodology. B.Tech. Thesis, National Institute of Technology Rourkela, India; 2011

17. Galantucci LM, Lavecchia F, Percoco G (2009) Experimental study aiming to enhance the surface finish of fused deposition modelled parts. CIRP Ann Manuf Technol 58:189-192. doi:10. 1016/j.cirp.2009.03.071

18. Kuo CC, Mao RC (2015) Development of a precision surface polishing system for parts fabricated by fused deposition modelling. Mater Manuf Process. doi:10.1080/10426914.2015. 1090594

19. Garg A, Anirban B, Ajay B (2016) On surface finish and dimensional accuracy of FDM parts after cold vapour treatment. Mater Manuf Processes 31(4):522-529. doi:10.1080/10426914. 2015.1070425

20. Priedeman WR, Smith DT. Smoothing method for layer manufacturing process. United States Patent No. US8123999B2, USA 2011 
21. Espalin D, Medina F, Arcaute K, Zinniel B, Hoppe T, Wicker R. Effects of vapour smoothing on ABS part dimensions. In: Proceedings of Rapid 2009 Conference and Exposition, May 12-14 2009, Schaumburg, USA. pp. 1-17

22. Stratasys Inc. Finishing touch smoothing station- service manual; 2010

23. ISO 4287:1997. Geometrical product specification (GPS)-surface texture: profile method-terms, definition and surface texture parameters

24. Boschetto A, Luana B (2015) Roughness prediction in coupled operations of fused deposition modeling and barrel finishing.
J Mater Process Technol 219:181-192. doi:10.1016/j.jmatprotec. 2014.12.021

25. UNI EN 20286-1: 1995. ISO system of limits and fits, bases of tolerances, deviations and fits

26. Devor RE, Chang T, Sutherland JW (2005) Statistical quality design and control contemporary concepts and methods. Pearson Prentice Hall (Second edition), New Jersey, pp 78-91 